\title{
ARTICLE OPEN Softening of phonon spectra in metallic glasses
}

\author{
Reza Mahjoub ${ }^{1}$, Nicholas E Hamilton ${ }^{1}$, Kevin $\mathrm{J} \mathrm{Laws}^{1}$ and Michael Ferry ${ }^{1}$
}

The vibrational spectra of a series of MgZnCa amorphous alloys were computed using density functional theory and implementing the small displacement method. The atomic structures of the alloys were obtained by $a b$ initio molecular dynamics simulations. The vibrational thermodynamic properties were calculated as a function of temperature and, in particular, the specific heat at low temperature was approximated by temperature cubed based on the Debye model. We computed the contribution of Mg vibrations to the specific heat and investigated the softening of $\mathrm{Mg}$ phonon spectra, where the maximum allowed vibrational frequency is lowered and highly collective diffusion processes are promoted. The statistical correlation between the reported critical casting thickness of the alloys and softening of $\mathrm{Mg}$ phonons was obtained. Similar calculations were performed for two distinctively different amorphous ZrTiCuAl alloys with large and small reported critical casting thickness, respectively. The findings were consistent with those of the MgZnCa alloys.

npj Computational Materials (2016) 2, 16029; doi:10.1038/npjcompumats.2016.29; published online 21 October 2016

\section{INTRODUCTION}

There has been increasing attention devoted to the effect of vibrational thermodynamics on the formation of different alloy phases and their associated phase transformations due to the improved accessibility of instruments capable of measuring and calculating the phonon frequencies. ${ }^{1}$ Various thermodynamic properties such as specific heat and vibrational entropy can be derived through the vibrational density of states (VDOSs). The VDOS obtained by molecular dynamics (MD) simulations has provided useful insight into how phonon spectra are affected by both the size and surface properties of silicon nanoparticles; this is of major significance in the design of nanodevices with better heat transport properties. It was found that, as the Si particles become smaller, the density of vibrational states increases at low frequencies, and modes are transferred from the high-frequency end to the intermediate range and transverse optical modes peak are shifted to higher frequencies. ${ }^{2}$

In metallic glasses, vibrational characteristics have also been used for explaining mechanical properties. For example, recent MD simulations of a $\mathrm{Cu}_{64} \mathrm{Zr}_{36}$ metallic glass showed that the strongest participation in the modes with lowest vibrational frequency and, hence, weakest spring constants (i.e., soft modes) originate predominantly from meagrely populated polyhedra. These soft spots provide fertile sites for accommodating shear transformations. $^{3}$

The softening of long wavelength acoustic phonons in $\mathrm{PdSiCu}$, ZrTiCuNiBe, CeAINiCu and ZrNbCuNiBe amorphous alloys relative to their crystalline phases have been observed experimentally using ultrasound measurements. ${ }^{4-6}$ Such observations are consistent with the low-temperature measurements of their specific heat, whereby an increase in temperature causes a steeper increase in specific heat in the amorphous alloys compared with their crystalline counterparts.

Specific heat is believed to be a fundamental property of materials and, in particular, glass-forming alloys. In undercooled metallic alloy liquids, the change in specific heat with temperature is experimentally ${ }^{7}$ and theoretically ${ }^{8}$ shown to correlate to the fragility of the liquid and glass-forming ability.

The VDOSs of amorphous systems obtained from atomistic simulations can be computed from the Fourier transform of the temporal autocorrelation of velocity, ${ }^{9}$ the linear response and small displacement methods. To calculate phonon spectra based on velocity autocorrelation by $a b$ initio MD simulation methods may not be practical, as the simulation time is either impractically long to equilibrate the system at low temperatures or to achieve a frequency resolution. ${ }^{10}$

Although increasing attention has been devoted to the study of vibrational modes in amorphous systems, $a b$ initio simulations have been lagging behind due to the complexity and resourceintensive nature of such calculations. In this work, density functional theory was used to implement the small displacement method for computing the phonon spectra of a series of $\mathrm{MgZnCa}$ amorphous alloys, of which the atomic configurations were also obtained by $a b$ initio MD simulations. We have examined the effect of composition on the VDOS and calculated the vibrational thermodynamic properties, particularly the contribution of the various constituent atom species to the specific heat, as a function of temperature. To broaden our study, a distinctly different system of ZrTiCuAl amorphous alloys was chosen and the calculations were repeated.

\section{RESULTS AND DISCUSSION}

Figure 1 gives the total VDOS for two of the alloys investigated $\left(\mathrm{Mg}_{68} \mathrm{Zn}_{28} \mathrm{Ca}_{4}\right.$ and $\left.\mathrm{Mg}_{72} \mathrm{Zn}_{24} \mathrm{Ca}_{4}\right)$; the critical casting thickness $\left(Z_{c}\right.$, below which a fully amorphous stricture is generated, Table 1 ) of these alloys are largest and smallest respectively. Further, the reduced VDOS, defined as $g(\omega) / \omega^{2}$ is depicted in the inset of Figure 1, where at low frequencies an enhancement in VDOS relative to the Debye squared-frequency law (the Boson Peak) is seen. ${ }^{11}$

Figure 2 shows the partial VDOS for the constituent atom species of these alloys with the inset showing a blow up of the $\mathrm{Mg}$

\footnotetext{
${ }^{1}$ School of Materials Science and Engineering, The University of New South Wales, Sydney, NSW, Australia. Correspondence: R Mahjoub (reza.mahjoub@unsw.edu.au)

Received 8 December 2015; revised 15 June 2016; accepted 21 July 2016 
partials. It is pertinent to note that for all alloys, the total and partial VDOS are asymmetric and positively skewed, where the distribution is concentrated on lower frequencies. However, specific heat is commonly used as an elaborate quantitative illustration of this shift to lower energies in the density of states. ${ }^{4}$

Further examination of partial VDOS indicates that the distribution of states depends markedly on atomic concentration. The peak of the partial VDOS is found to be inversely correlated to concentration of the respective species, as shown in Figure $3 a, b$ for $\mathrm{Mg}$ and $\mathrm{Zn}$, respectively. In addition, the area under the partial VDOS representing total available vibrational states per atom are shown as a function of $\mathrm{Mg}$ and $\mathrm{Zn}$ concentration in Figure $3 \mathrm{c}, \mathrm{d}$, respectively. It is seen that, as the concentration of each species increases, there is a decrease in the total available states. As such, $\mathrm{Ca}$ that has the smallest concentration in each alloy, has the highest vibrational states per atom followed by $\mathrm{Zn}$ and $\mathrm{Mg}$.

The temperature dependence of specific heat at low temperatures is of particular importance in the analysis of physical properties of solids, as it reveals ionic and electronic contributions to the thermal energy. Figure $4 a$ illustrates the contribution of $\mathrm{Mg}$ to the specific heat of the $\mathrm{Mg}_{72} \mathrm{Zn}_{24} \mathrm{Ca}_{4}$ and $\mathrm{Mg}_{68} \mathrm{Zn}_{28} \mathrm{Ca}_{4}$ alloys over the temperature range $\sim 0-30 \mathrm{~K}$. According to the Debye approximation, the contribution of phonons to the specific heat is proportional to the cube of temperature $(T)$ at low absolute temperatures $^{12}$ such that the specific heat can be expressed as $C_{V} \approx \beta T^{3}$, where $\beta=12 \pi^{4} R / 5 \theta_{\mathrm{D}}^{3}$, and $R$ and $\theta_{\mathrm{D}}$ are the gas constant and Debye temperature, respectively. ${ }^{13}$ The Debye approximation

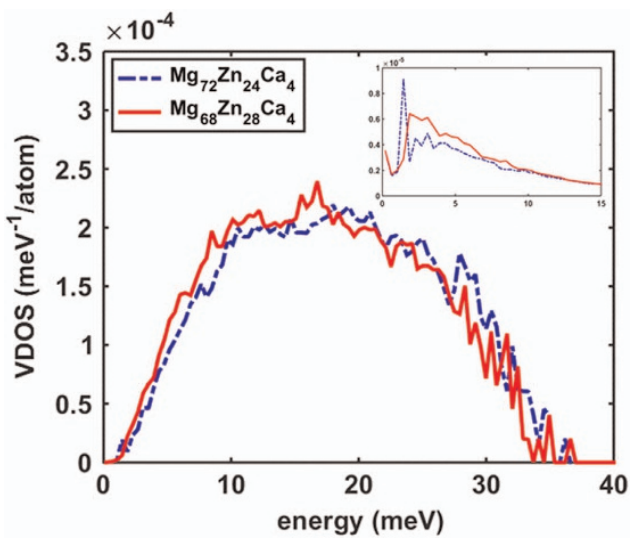

Figure 1. Total VDOS of $\mathrm{Mg}_{68} \mathrm{Zn}_{28} \mathrm{Ca}_{4}$ and $\mathrm{Mg}_{72} \mathrm{Zn}_{24} \mathrm{Ca}_{4}$ alloys. The inset shows the reduced VDOS of these two alloys. was suggested to evaluate analytically the specific heat based on the quantum theory of harmonic crystal and predicted the temperature cube law for $C_{V}$, which agreed very well with the observation at very low temperatures. The approximation was based on the fact that, at very low temperatures, the contribution to $C_{V}$ of modes with $\hbar \omega \gg k_{B} T$ is negligible, whereas for acoustic modes with sufficiently long wavelength, the contribution to $C_{V}$ is appreciable and the corresponding vibrations can be regarded as linearly dispersed. ${ }^{12}$ At very low temperatures, not only are such simplifications valid for amorphous solids but also the measurements of specific heat of glassy solids agree well with the Debye temperature cube law. $4,5,13$

The energy at $\theta_{D}$ is related to the Debye frequency $\omega_{D}$ by $k_{\mathrm{B}} \theta_{\mathrm{D}}=\hbar \omega_{\mathrm{D}}$ and $\omega_{\mathrm{D}}$ represents the maximum allowed vibrational frequency in the structure. Both $\theta_{\mathrm{D}}$ and $\omega_{\mathrm{D}}$ may also be regarded as a measure of the stiffness of a material, ${ }^{12}$ as they are related to the curvature of the energy landscape at the local minimum of a basin (stiffness). ${ }^{14}$ Assuming that the contribution of electrons to the specific heat in an electrical conductor is proportional to $T$, then it can be shown that $C_{V} / T=\beta T^{2}+\gamma$, where $\beta$ and $\gamma$ are the gradient and intercept of $C_{\mathrm{V}} / T$ as a function of $T^{2}$. Table 1 provides $\beta$ and $\gamma$ as obtained from the contributions of $\mathrm{Mg}$ and $\mathrm{Zn}$ to the specific heat at low temperatures. The selected temperature range was in the range $5-20 \mathrm{~K}$ to avoid pronounced temperature dependence of $\beta$ and hence $\theta_{\mathrm{D}}$. The values of $\beta$ show that some alloys are softer than the others and further investigation of the calculated and experimentally measured properties reveals that there is a statistical correlation between $\beta_{\mathrm{Mg}}$ and the critical

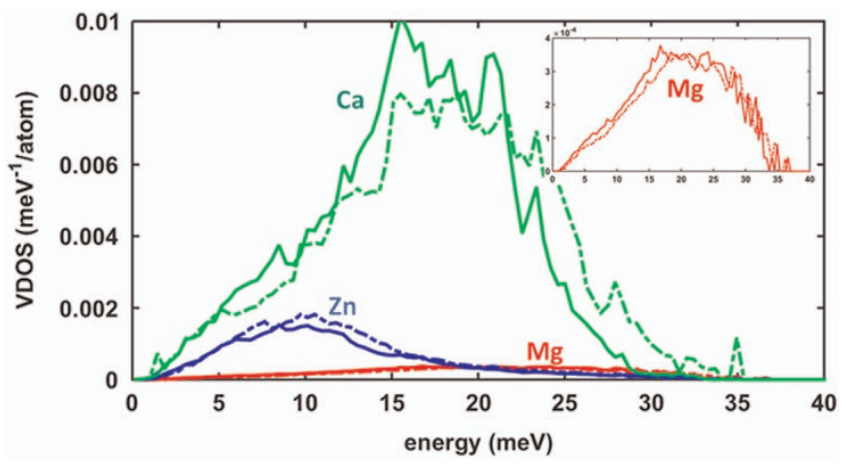

Figure 2. Partial VDOS of $\mathrm{Mg}_{68} \mathrm{Zn}_{28} \mathrm{Ca}_{4}$ (solid line) and $\mathrm{Mg}_{72} \mathrm{Zn}_{24} \mathrm{Ca}_{4}$ (broken-dotted line) alloys. The inset shows the contribution of $\mathrm{Mg}$ atoms to VDOS.

\begin{tabular}{|c|c|c|c|c|c|c|c|c|c|c|c|}
\hline $\mathrm{Mg}_{72} \mathrm{Zn}_{24} \mathrm{Ca}_{4}$ & $1.92 \mathrm{E}+01$ & $3.53 \mathrm{E}-04$ & $6.94 \mathrm{E}-03$ & $1.43 \mathrm{E}-05$ & $7.060 \mathrm{E}-04$ & $1.05 \mathrm{E}+01$ & $1.82 \mathrm{E}-03$ & $2.08 \mathrm{E}-02$ & $5.68 \mathrm{E}-05$ & $2.430 \mathrm{E}-03$ & 1.1 \\
\hline $\mathrm{Mg}_{70} \mathrm{Zn}_{26} \mathrm{Ca}_{4}$ & $2.38 \mathrm{E}+01$ & $3.48 \mathrm{E}-04$ & $7.14 \mathrm{E}-03$ & $1.43 \mathrm{E}-05$ & 8.394E-04 & $1.05 \mathrm{E}+01$ & $1.61 \mathrm{E}-03$ & $1.92 \mathrm{E}-02$ & $5.86 \mathrm{E}-05$ & $2.483 \mathrm{E}-03$ & 2.8 \\
\hline $\mathrm{Mg}_{68} \mathrm{Zn}_{28} \mathrm{Ca}_{4}$ & $1.67 \mathrm{E}+01$ & $3.78 \mathrm{E}-04$ & $7.35 \mathrm{E}-03$ & $1.66 \mathrm{E}-05$ & 8.393E-04 & $8.48 \mathrm{E}+00$ & $1.52 \mathrm{E}-03$ & $1.79 \mathrm{E}-02$ & $6.14 \mathrm{E}-05$ & 4.034E-03 & 4.0 \\
\hline $\mathrm{Mg}_{70} \mathrm{Zn}_{24} \mathrm{Ca}_{6}$ & $2.01 \mathrm{E}+01$ & $3.46 \mathrm{E}-04$ & $7.14 \mathrm{E}-03$ & 1.47E-05 & $6.165 \mathrm{E}-04$ & $7.65 \mathrm{E}+00$ & $1.80 \mathrm{E}-03$ & $2.08 \mathrm{E}-02$ & $5.76 \mathrm{E}-05$ & $2.286 \mathrm{E}-03$ & 3.0 \\
\hline $\mathrm{Mg}_{68} \mathrm{Zn}_{26} \mathrm{Ca}_{6}$ & $2.30 \mathrm{E}+01$ & $3.58 \mathrm{E}-04$ & 7.35E-03 & $1.52 \mathrm{E}-05$ & $8.411 \mathrm{E}-04$ & $8.89 \mathrm{E}+00$ & $1.74 \mathrm{E}-03$ & $1.92 \mathrm{E}-02$ & $5.55 \mathrm{E}-05$ & $1.548 \mathrm{E}-03$ & 3.3 \\
\hline $\mathrm{Mg}_{66} \mathrm{Zn}_{28} \mathrm{Ca}_{6}$ & $2.21 \mathrm{E}+01$ & $3.75 \mathrm{E}-04$ & $7.58 \mathrm{E}-03$ & $1.51 \mathrm{E}-05$ & $1.423 \mathrm{E}-03$ & $9.72 \mathrm{E}+00$ & $1.64 \mathrm{E}-03$ & $1.79 \mathrm{E}-02$ & $6.00 \mathrm{E}-05$ & $4.253 \mathrm{E}-03$ & 2.8 \\
\hline $\mathrm{Mg}_{64} \mathrm{Zn}_{30} \mathrm{Ca}_{6}$ & $2.34 \mathrm{E}+01$ & $3.84 \mathrm{E}-04$ & $7.81 \mathrm{E}-03$ & $1.39 \mathrm{E}-05$ & 1.193E-03 & $1.01 \mathrm{E}+01$ & $1.46 \mathrm{E}-03$ & $1.67 \mathrm{E}-02$ & $5.50 \mathrm{E}-05$ & $2.404 \mathrm{E}-03$ & 2.7 \\
\hline $\mathrm{Mg}_{62} \mathrm{Zn}_{32} \mathrm{Ca}_{6}$ & $2.38 \mathrm{E}+01$ & 4.12E-04 & 8.06E-03 & $1.32 \mathrm{E}-05$ & $6.733 \mathrm{E}-04$ & $1.01 \mathrm{E}+01$ & $1.26 \mathrm{E}-03$ & $1.56 \mathrm{E}-02$ & 5.27E-05 & $2.293 \mathrm{E}-03$ & 1.5 \\
\hline
\end{tabular}

Abbreviations: VDOS, vibrational density of state.

Units are meV for the frequency of maximum VDOS $f_{\text {mode }}$ Number/meV/atom for the maximum of partial vibrational density of states VDOS max. Number/atom for the partial VDOS integral $I . \mathrm{k}_{\mathrm{B}} / \mathrm{K}^{3} /$ atom for the gradient of $C_{\mathrm{v}} / T$ as a function of $T^{2}, \beta . \mathrm{k}_{\mathrm{B}}^{2} / K / \mathrm{atom}$ for the intercept of $C_{\mathrm{v}} / T$ as a function of $T^{2}, \gamma$. $\mathrm{mm}$ for critical casting thickness $Z_{c}$. 

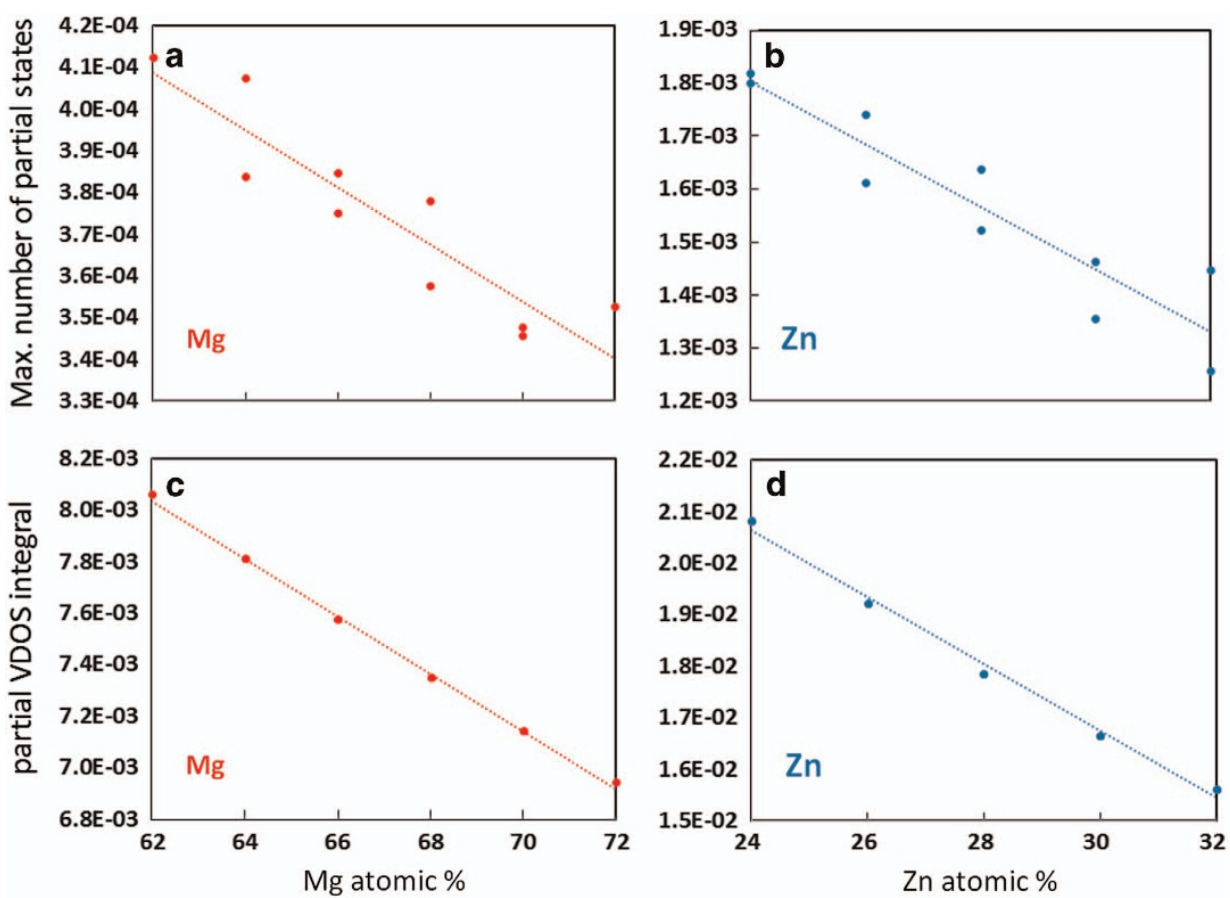

Figure 3. (a, b) Maximum number of partial vibrational states and (c, $\mathbf{d})$ the integral of the partial VDOS per atom over all frequencies as a function of $\mathrm{Mg}$ and $\mathrm{Zn}$ concentration, respectively.

a

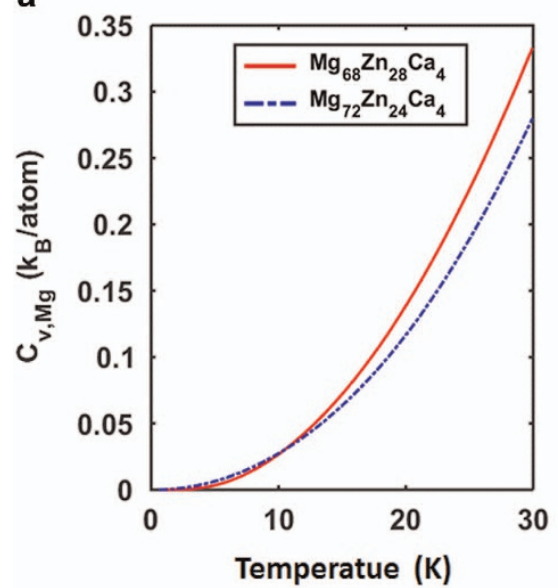

b

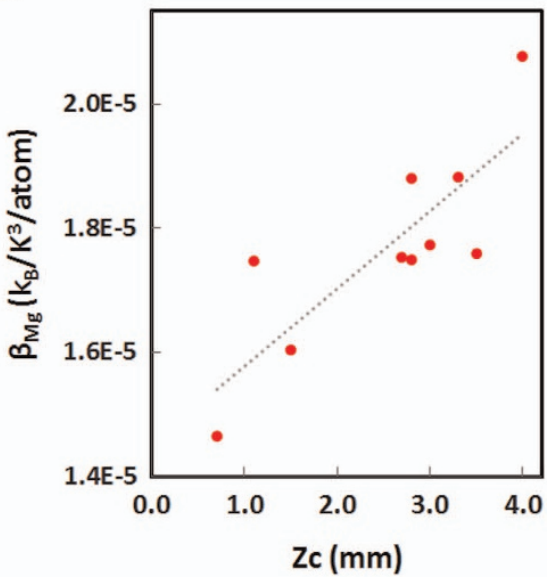

Figure 4. (a) Contribution of $\mathrm{Mg}$ vibrations to the specific heat for $\mathrm{Mg}_{72} \mathrm{Zn}_{24} \mathrm{Ca}_{4}$ and $\mathrm{Mg}_{68} \mathrm{Zn}_{28} \mathrm{Ca}_{4}$ alloys. (b) $\beta_{\mathrm{Mg}}$ versus critical casting thickness $\left(Z_{c}\right)$. The coefficient of determination for the linear fit is 0.68 .

casting thickness $\left(Z_{c}\right)_{1}^{15}$ as depicted in Figure $4 b$. In other words, as the temperature is raised, the contribution of $\mathrm{Mg}$ phonons to the specific heat tends to show a steeper increase in those alloys with greater $Z_{c}$. As $\beta$ is inversely proportional to both $\theta_{D}$ and $\omega_{D}$, a greater $Z_{c}$ concurs with a lower Debye frequency of Mg atoms vibrating in the vicinity of a local minimum in a shallow megabasin of the energy landscape.

The energy landscape paradigm articulates that, for a material system comprising a certain number of particles in a given volume, the energy landscape is fixed, whereas the process by which the landscape is sampled depends on temperature. ${ }^{16,17}$ In the supercooled liquid at temperatures higher than the glass transition $\left(T_{\mathrm{g}}\right)$, configurational sampling of neighbouring megabasins occurs ${ }^{17}$ and megabasins with smaller curvature at their local minimum implying softened phonon spectra may notably promote a highly cooperative diffusion process where a large number of atoms (typically above 10 (ref. 18)) take part in a collective hopping over energy barriers. The collective diffusion in supercooled liquids has been observed both experimentally ${ }^{19}$ and theoretically in MD simulations. ${ }^{20}$ At lower temperatures, hopping over barriers in the energy landscape requires large atomic amplitudes that can be provided by localised soft vibrations. ${ }^{18}$ At higher temperatures, there is an increase in both the atomic displacements and the number of atoms participating in the jumps. $^{21}$

It is pertinent to note that, although crystallisation is universally known to be a first-order thermodynamic transformation, ${ }^{22,23}$ glass transition has been prevailingly considered as a largely dynamical phenomenon ${ }^{24}$ and soft phonons are known to be related to the dynamics of amorphous alloys. ${ }^{18,25}$

Although the energy landscape curvature and mean-squared atomic displacements (MSD) are two different quantities, they are inter-connected often in complicated relations. The calculated MSD of Mg atoms in the vicinity of glass transition temperature ${ }^{15}$ 
$(400 \mathrm{~K})$ for the alloys with the largest $\left(\mathrm{Mg}_{68} \mathrm{Zn}_{28} \mathrm{Ca}_{4}\right)$ and smallest $Z_{c}\left(M_{64} Z_{32} \mathrm{Ca}_{4}\right)$ are depicted in Figure 5 . It is seen that the dynamic slows down for alloy the $Z_{c}$ of which is larger. To minimise the fluctuations in the MSD plots and to provide a clearer perspective, the alloys have been categorised by their reported $Z_{\mathrm{c}}$ as small $Z_{\mathrm{c}}$ (with $Z_{\mathrm{c}} \leqslant 2 \mathrm{~mm}$ ), medium (with $2 \mathrm{~mm}<Z_{c} \leqslant 3 \mathrm{~mm}$ ) and large $Z_{c}$ (with $Z_{c}>3 \mathrm{~mm}$ ), respectively. The mean MSD of Mg for each group at $400 \mathrm{~K}$ has been calculated and provided in the inset to Figure 5 . It is observed that the dynamic slowdown is most prominent for the category with large $Z_{c}$ followed by alloys with medium and small $Z_{c}$. It should be noted that $M g_{70} Z n_{26} C a_{4}$ was excluded from the medium $Z_{c}$ and considered as an outlier.

To broaden the variety of samples and strengthen the validity of the correlation, we have opted for a distinctively different family of alloys and applied the same method to compute the vibrational properties of amorphous $\mathrm{Zr}_{61} \mathrm{Ti}_{2} \mathrm{Cu}_{25} \mathrm{Al}_{12}$ and $\mathrm{Zr}_{63.5} \mathrm{Ti}_{4.5} \mathrm{Cu}_{23} \mathrm{Al}_{9}$ structures.

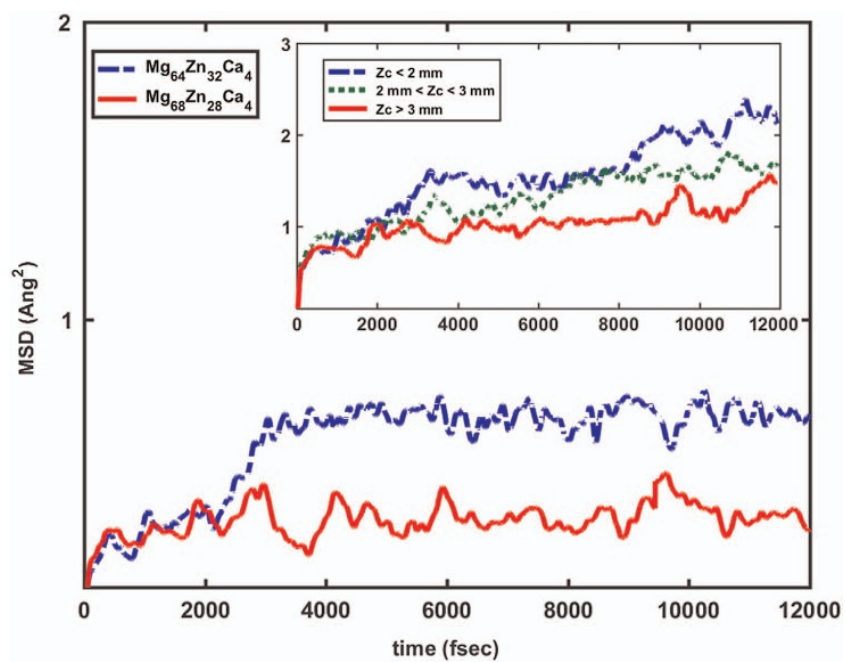

Figure 5. The mean-squared displacement (MSD) of Mg atoms in the vicinity of glass transition temperature with the largest $\left(M_{68} Z_{28} \mathrm{Ca}_{4}\right)$ and smallest $Z_{c}\left(M g 64 \mathrm{Zn}_{32} \mathrm{Ca}_{4}\right)$. The inset shows the averaged MSD of $\mathrm{Mg}$ for alloys with small, medium and large $Z_{\mathrm{c}}$, respectively.
The reported critical casting thickness for these alloys is 10 and $3 \mathrm{~mm}$, respectively. ${ }^{26,27}$ The partial VDOS of these alloys representing $\mathrm{Cu}$ vibrational spectra are shown in Figure $6 \mathrm{a}$. Figure $6 \mathrm{~b}$ depicts the contribution of $\mathrm{Cu}$ vibrations to the specific heat of these $\mathrm{Zr}$-based alloys as a function of temperature. Having fitted the linear regression line to $C_{\mathrm{V}} / T$ as a function of $T^{2}$, we find $\beta_{\mathrm{Cu}}$ to be 0.02195 and $0.02063 \mathrm{k}_{\mathrm{B}} / \mathrm{K}^{3}$ /atom for $\mathrm{Zr}_{61} \mathrm{Ti}_{2} \mathrm{Cu}_{25} \mathrm{Al}_{12}$ and $\mathrm{Zr}_{63.5} \mathrm{Ti}_{4.5} \mathrm{Cu}_{23} \mathrm{Al}_{9}$, respectively. As for the contribution of $\mathrm{Al}$ vibrations to the specific heat, $\beta_{\mathrm{Al}}$ was computed to be 0.00606 and $0.00586 k_{B} / K^{3} /$ atom for the former and the latter alloy, respectively. It is also seen that here a higher $Z_{c}$ concurs with a greater $\beta_{\mathrm{Cu}}$ and $\beta_{\mathrm{Al}}$. Indeed, the analysis of specific heat in $\mathrm{MgZnCa}$ amorphous alloys appears to be consistent to that of these widely dissimilar ZrTiCuAl metallic glasses. Although $\mathrm{Cu}$ concentration is less than half of $\mathrm{Zr}$, the mobility of $\mathrm{Cu}$ was calculated and found to be the highest, as shown in Figure $6 c$.

\section{MATERIALS AND METHODS}

First-principles MD was used to simulate the melting and quenching processes of the alloys given in Table $1 .^{28}$ We considered 200 atoms in a cell with dimensions of the order of $\sim 18 \AA$. The software of choice was $V_{\text {VASP }}^{29}$ implementing the projector augmented wave method to represent core electrons. ${ }^{30}$ Generalised gradient functional was used to approximate the exchange and correlation energies. ${ }^{31}$ To sample the Brillouin zone, $\Gamma$ point only was considered due to the large cell size. The systems were equilibrated well above the liquidus temperature and then quenched to room temperature with a cooling rate of $0.66 \times 10^{13} \mathrm{~K} / \mathrm{s}$ at 3,000 time steps per $100 \mathrm{~K}^{32}$ The simulated pair distribution functions were found to be in close agreement with the results of high-energy X-ray scattering experiments. ${ }^{28}$

To calculate the vibrational properties, the k-point sampling was increased to $2 \times 2 \times 2$, a full grid was adopted for Fourier transformation, the energy cut off was raised to $30 \%$ above the default values and the equilibrated configuration at room temperature was relaxed using the conjugate gradient method until the force on each atom was below $0.01 \mathrm{eV} / \AA$ and the total drift in forces were minimal. At this stage, the system is located at a local minimum and the energy can be regarded as the equilibrium potential energy. To find the Taylor expansion of the energy, the small displacement method was implemented ${ }^{33}$ on the relaxed structure, displacing each configurational degree of freedom by $0.02 \AA$. Six hundred different configurations thus created were subject to static calculations based on density functional theory and the force field for each displacement was used to calculate the dynamical matrix and its eigenvalues, the square roots of which constitute the vibrational frequencies. ${ }^{1}$ The total free energy of the system can be approximated a

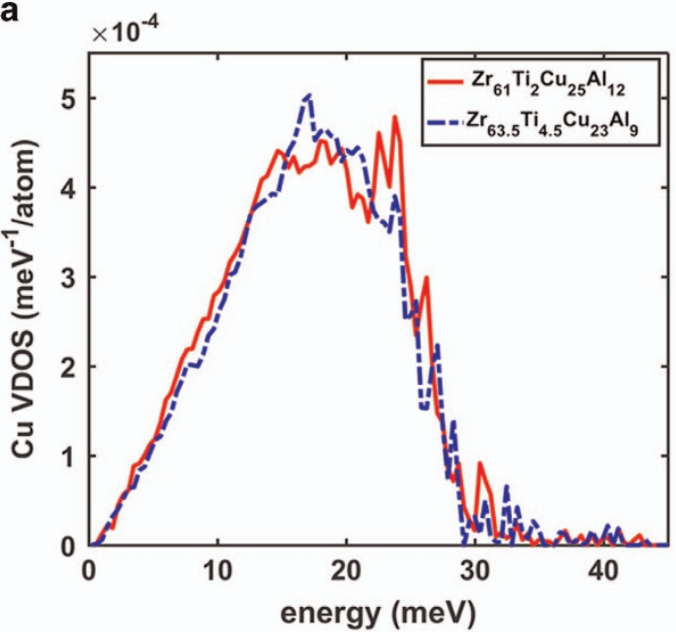

b

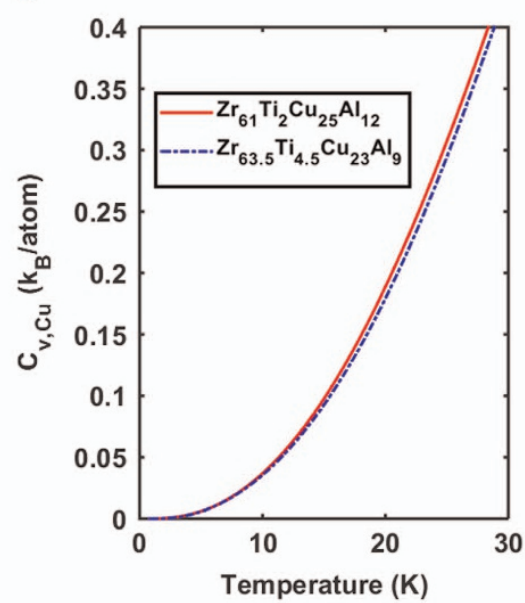

C

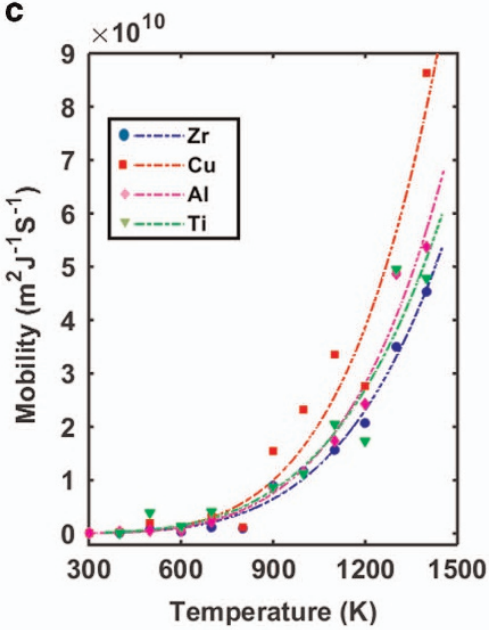

Figure 6. (a) Partial VDOS of $\mathrm{Zr}_{61} \mathrm{Ti}_{2} \mathrm{Cu}_{25} \mathrm{Al}_{12}$ (solid line) and $\mathrm{Zr}_{63.5} \mathrm{Ti}_{4.5} \mathrm{Cu}_{23} \mathrm{Al}_{9}$ (broken-dotted line) representing the contribution of $\mathrm{Cu}$ atoms to VDOS. (b) Contribution of $\mathrm{Cu}$ vibrations to the specific heat and (c) the mean mobility of atom species (broken-dotted lines are drawn as guides to the eye), as a function of temperature for $\mathrm{Zr}_{61} \mathrm{Ti}_{2} \mathrm{Cu}_{25} \mathrm{Al}_{12}$ and $\mathrm{Zr}_{63.5} \mathrm{Ti}_{4.5} \mathrm{Cu}_{23} \mathrm{Al}_{9}$ alloys, respectively. 
by the sum of the configurational energy of the relaxed structure and the total energy of the harmonic oscillators. ${ }^{33}$ The latter is expressed $a^{13}$ $F_{\text {harm }}=\int g(\omega)<n(T)>d \omega$, where $\omega, T,<n(T)>$ and $g(\omega)$ are the vibrational frequency, temperature, Plank distribution and VDOS, respectively, which can be obtained from the square roots of the eigenvalues of the dynamical matrix. Once the vibrational free energy is known, the vibrational entropy and internal energy can also be calculated. In particular, the specific heat at constant volume can be obtained from $C_{V}=\partial F_{\text {harm }} / \partial T$.

\section{ACKNOWLEDGEMENTS}

We acknowledge financial support from the Australian Research Council (ARC) for funding of this work via the ARC Centre of Excellence for Design in Light Metals (CE0561574) and ARC Discovery grants scheme (DP120102863). Computational resources were provided by Intersect Australia Ltd and the computation was undertaken on the $\mathrm{NCI}$ National Facility in Canberra, Australia, which is supported by the Australian Commonwealth Government.

\section{CONTRIBUTIONS}

RM carried out the $a b$ initio MD and density functional theory calculations for $\mathrm{MgZnCa}$ alloys. $\mathrm{NH}$ carried out the $a b$ initio MD and density functional theory calculations for $\mathrm{ZrTiCuAl}$ alloys. $\mathrm{KL}$ designed the experimental programme and MF coordinated the overall project. All authors contributed to the interpretation of the results and to the writing of the paper.

\section{COMPETING INTERESTS}

The authors declare no conflict of interest.

\section{REFERENCES}

1. Fultz, B. Vibrational thermodynamics of materials. Prog. Mater. Sci. 55, 247-352 (2010).

2. Meyer, R. \& Comtesse, D. Vibrational density of states of silicon nanoparticles. Phys. Rev. B 83, 014301(1-6) (2011).

3. Ma, E.van Tuning order in disorder. Nat. Mater. 14, 547-552 (2015).

4. Golding, B., Hsu, F. S. L. \& Bagley, B. G. Soft transverse phonons in a metallic glass. Phys. Rev. Lett. 29, 68 (1972).

5. Wang, W. H., Bai, H. Y., Luo, J. L., Wang, R. J. \& Jin, D. Supersoftening of transverse phonons in Zr41Ti14Cu12.5Ni10Be22.5 bulk metallic glass. Phys. Rev. B 62 $25-28(2000)$.

6. Zhang, Y., Zhao, D. Q., Wang, R. J. \& Wang, W. H. Formation and properties of Zr48Nb8Cu14Ni12Be18 bulk metallic glass. Acta Mater. 51, 1971-1979 (2003).

7. Busch, R., Schroers, J. \& Wang, W. H. Thermodynamics and kinetics of bulk metallic glass. MRS Bull 32, 620-623 (2007).

8. Ding, J., Cheng, Y.-Q., Sheng, H. \& Ma, E. Short-range structural signature of excess specific heat and fragility of metallic-glass-forming supercooled liquids. Phys. Rev. B 85, 060201(1-5) (2012).

9. Rahman, A. Correlations in mothion of atoms in liquid argon. Phys. Rev. 136, A405 (1964).

10. Baroni, S., de Gironcoli, S., Dal Corso, A. \& Giannozzi, P. Phonons and related crystal properties from density-functional perturbation theory. Rev. Mod. Phys. 73 515-562 (2001)

11. Guerdane, M. \& Teichler, H. Short-range-order lifetime and the "boson peak" in a metallic glass model. Phys. Rev. Lett. 101, 065506(1-4) (2008).

12. Ashcraft, N. W. \& Mermin, N. D. Solid State Physics (Harcourt: Orlando, FL, USA, 1976).
13. Kittel, C. Introduction to Solid State Physics. 8th edn (Wiley: New York, USA, 2004).

14. Ding, J., Patinet, S., Falk, M. L., Cheng, Y. \& Ma, E. Soft spots and their structural signature in a metallic glass. Proc. Nati Acad. Sci. USA 111, 14052-14056 (2014).

15. Cao, J. D., Laws, K. J., Birbilis, N. \& Ferry, M. Potentiodynamic polarisation study of bulk metallic glasses based on the Mg-Zn-Ca ternary system. Corros. Eng. Sci. Technol. 47, 329-334 (2012).

16. Goldstei, M. Viscous liquids and glass transition-A potential energy barrier picture. J. Chem. Phys. 51, 3728 (1969).

17. Debenedetti, P. G. \& Stillinger, F. H. Supercooled liquids and the glass transition. Nature 410, 259-267 (2001).

18. Faupel, F. et al. Diffusion in metallic glasses and supercooled melts. Rev. Mod. Phys. 75, 237-280 (2003)

19. Ehmler, H., Heesemann, A., Ratzke, K., Faupel, F. \& Geyer, U. Mass dependence of diffusion in a supercooled metallic melt. Phys. Rev. Lett. 80, 4919-4922 (1998).

20. Schober, H. R. Soft phonons in glasses. Physica A 201, 14-24 (1993).

21. Oligschleger, C. \& Schober, H. R. Collective jumps in a soft-sphere glass. Phys. Rev. B 59, 811-821 (1999).

22. Kelton, K. F. \& Gear, A.L. Nucleation in Condensed Matter (Elsevier: Amsterdam, 2010).

23. Mahjoub R.. et al.. Heterogeneous nucleation at inoculant particles in a glass forming alloy: an $a b$ initio molecular dynamics investigation of interfacial properties and local chemical bonding. Comput. Mater. Sci. 108, 94-102 (2015).

24. Royal, C. P. \& Williams, S. R. The role of local structure in dynamical arrest. Phys. Rep. 560, 1-75 (2015).

25. Zhang, Y. et al. Strong correlations of dynamical and structural heterogeneities with localized soft modes in a Cu-Zr metallic glass. Appl. Phys. Lett. 105 (2014).

26. He, Q. \& Xu, J. Locating malleable bulk metallic glasses in $\mathrm{Zr}$-Ti-Cu-Al alloys with calorimetric glass transition temperature as an indicator. J. Mater. Sci. Technol. 28 1109-1122 (2012).

27. He, Q. A., Cheng, Y. Q., Ma, E. \& Xu, J. A. Locating bulk metallic glasses with high fracture toughness Chemical effects and composition optimization. Acta Mater. 59, 202-215 (2011).

28. Mahjoub, R., Laws, K. J., Scicluna, J. P., Daniels, J. E. \& Ferry, M. A first principles molecular dynamics study of the relationship between atomic structure and elastic properties of Mg-Zn-Ca amorphous alloys. Comput. Mater. Sci. 96, 246-255 (2015).

29. Kresse, G. \& Furthmuller, J. Efficient iterative schemes for ab initio total-energy calculations using a plane-wave basis set. Phys. Rev. B 54, 11169-11186 (1996).

30. Kresse, G. \& Joubert, D. From ultrasoft pseudopotentials to the projector augmented-wave method. Phys. Rev. B 59, 1758-1775 (1999).

31. Perdew, J. P., Burke, K. \& Ernzerhof, M. Generalized gradient approximation made simple. Phys. Rev. Lett. 77, 3865-3868 (1996).

32. Senkov, O. N. et al. Atomic structure of Ca40+XMg25Cu35-X metallic glasses J. Appl. Phys. 111, 123515 (2012).

33. Alfe, D. PHON: a program to calculate phonons using the small displacement method. Comput. Phys. Commun. 180, 2622-2633 (2009).

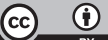

This work is licensed under a Creative Commons Attribution 4.0 International License. The images or other third party material in this article are included in the article's Creative Commons license, unless indicated otherwise in the credit line; if the material is not included under the Creative Commons license, users will need to obtain permission from the license holder to reproduce the material. To view a copy of this license, visit http://creativecommons.org/licenses/ by/4.0/

(c) The Author(s) 2016 